\title{
Safe Nasoendoscopy Assisted Procedure in the Post COVID-19 Pandemic Era
}

\author{
Ajith George $^{1}$, Christopher Coulson ${ }^{2}$, and Mark Prince ${ }^{3}$ \\ ${ }^{1}$ Keele University \\ ${ }^{2}$ Queen Elizabeth Hospital Birmingham \\ ${ }^{3}$ Aston University
}

May 6, 2020

\begin{abstract}
NA

For centuries it has been humankind's instinct to cover the mouth and nose when coughing or sneezing. Common sense would dictate this instinctively reduces the dispersion of aerosol and droplets and thus the spread of contact and airborne infections.
\end{abstract}

Aerosol generating procedures (AGPs) have become a new byword for procedures that put clinicians at increased risk of contracting COVID-19. Whilst the title suggests the risk is simply in aerosols, the science is much more interesting. Droplets and aerosols are different, with the distinction between them based on size. Whilst experts disagree on the absolute size when an aerosol becomes a droplet, the general acceptance is that anything bigger than 50 microns $(0.05 \mathrm{~mm})$ is a droplet and those smaller are aerosols.

In most contagious respiratory infections, the principal transmission agents are droplets (1). This is due to the relatively high viral load in a droplet, purely due to its large size, and also the fact that large droplets have weight, and so gravity pulls them down onto surfaces that others can touch - so passing it on. This is why washing hands is so effective against droplet spread.

Aerosol transmission is thought to be a much less frequent cause of transmission, mainly due to the very small viral load (given the aerosol itself is by definition very small). However, it is clearly more concerning as these very small, and therefore very light particles, can travel large distances on air currents and can be directly inhaled. That said, it is thought to only play a minor role in transmission compared to droplet spread.

During the COVID-19 pandemic PHE (Public Health England) updated guidance on what it considers (AGPs) Aerosol Generating Procedures. Included within this list were examinations of the upper aerodigestive tract in ENT. Any procedure enacting air over a fluid mucosal surface therefore poses a risk of viral dispersion within both droplets and aerosol. Healthcare workers were recommended to reduce endoscopy of the nose and throat. Any essential examination had to be performed using high level PPE including an respirator (N95 or FFP3)(2).

Anfinrud et al (3) graphically represented a visual reduction in aerosol production by creating light sheet from a 532-nm green LASER. Comparisons were made between a person talking with and without a cover for the mouth, in their instance, a slightly dampened wash cloth. Light flashes were recorded to evaluate 
the number of droplets ranging between 5-200 microns. They showed that by covering the mouth, virtually no light flashes were seen. This observation supports the well-known concept that covering the mouth does indeed reduce droplet production.

On impact with smooth surfaces droplets disperse to smaller sizes and can aerosolise. Similarly impact onto soft surfaces absorbs droplets reducing their projection as well as the tendency to aerosolise (3).

As the pandemic plateaus in countries across the world various strategies are to be considered to return to a new normal. This would facilitate the resumption of diagnostic services whilst maintaining the protection to healthcare workers. One suggestion is the use of facemasks to help reduce the risk of inadvertent droplet dispersion (2). Despite the 'soft surface' barrier masks create, in the ENT setting, facemasks obscure access to the nasal cavity thus preventing nasoendoscopy.

The 'SNAP' (Safe Nasoendoscopic Airway Procedure) developed by endoscope-i Ltd (West Midlands, UK) is a single-use, valved endoscopic port, retrofitted to any surgical mask (Figure 1), permitting entry of a $4 \mathrm{~mm}$ flexible and rigid endoscope to examine the naso and pharyngolarynx. The valve, a $10.9 \mathrm{~mm}$ cylindrical tube truncated by two opposing 45 degree inclined membranes 700 microns thick, approaches a point but terminates in a 700 micron thick and 500 micron wide plateau, creating a 'duck bill' formation The valves are formed using a FFF (fused filament fabrication) 3D printing technique with a Flashforge Creator Pro 3D printer. The plateau atop the valve serves to provide a reference for introducing a slit using a hardened steel razor blade that is 400 microns thin. The blade is mounted in a jig to ensure angle, penetration depth and position are controlled as it is driven through the membrane. These measures ensure that the valve opening is observably consistent and less than 50 microns. Once the SNAP is fitted to a surgical mask, any cough or sneeze generated during the procedure is caught within the mask. The valve is configured such that pressure from the patient side will serve to collapse the walls of the valve membrane thereby further sealing the slit in the valve. This seal has been in vitro tested with aerosolised fluorescein(figure 2). The 45-degree angle of the valve walls from the non-patient side similarly allows the blunt tip of the nasoendoscope to deform the valve walls with ease. The cylindrical form of the walls encourages the valve membranes to return to their original flat shape following withdrawal of the endoscope.

During the COVID-19 pandemic our tertiary head and neck cancer referral centre managed 120 urgent $2 \mathrm{ww}$ cases. Using the Tikka et al calculator (4) $40 \%$ of referrals were redirected back to the GP. The remaining $60 \%$ either went direct for imaging or underwent endoscopy. In total 40 cases were endoscoped, 9 of which using the SNAP. All 9 cases scoped with the SNAP were completed without any adverse effect. No cough or sneeze was elicited during any of the examinations and observations between the two groups were identical. Subsequently one consultant lead FEES examination was performed under controlled conditions. Again, the procedure was completed without any complications. The patient self-remarked on the comfort of the endoscopy as a result of the stability provided by the SNAP device in the alar region the prevented inadvertent movement during the chin tuck and head turn exercises.

Our observations demonstrate the SNAP device is a practical and safe tool to aid reduction in droplet dispersion whilst performing nasoendoscopy. We hope to see the inclusion of such a device in recovery guidelines by national bodies in order to facilitate the return of safe nasoendoscopy in the post COVID Pandemic era.

\section{References}

1 Siegel J,Rhinehart E, Jackson M et al. 2007 Guideline for isolation precautions: Preventing Transmissions for infectious agents in Healthcare Settings. Am J Infect Control 2007; 35: S65-164

2 Rameau A, Young V, Amin M et al. Flexible Laryngoscopy and COVID-19. Otolaryngol Head Neck Surg 2020 DOI: $10.1177 / 0194599820921395$

3- Howland C, Antkowiak A, Castrejon-Pita R et al. Its harder to splash on soft solids. Phy Rev. Lett 117, 184502- Published 2016 
4- Tikka T, Pracy P, Paleri V. Refining the head and neck cancer referral guidelines: a two-centre analysis of 4715 referrals. Clin Otolaryngol. 2016 Feb;41(1):66-75. doi: 10.1111/coa.12597

\section{Legends}

Figure 1- The SNAP mask in use on a patient during flexible laryngoscopy (permission granted for publication)

Figure 2- In Vitro Aerosolised Fluorescein test results $1 \mathrm{ml} / \mathrm{s}$ through a 50 micro point comparing a mask fitted with SNAP with no mask and mask without mask. No visual difference seen and no leak though the SNAP valve. 


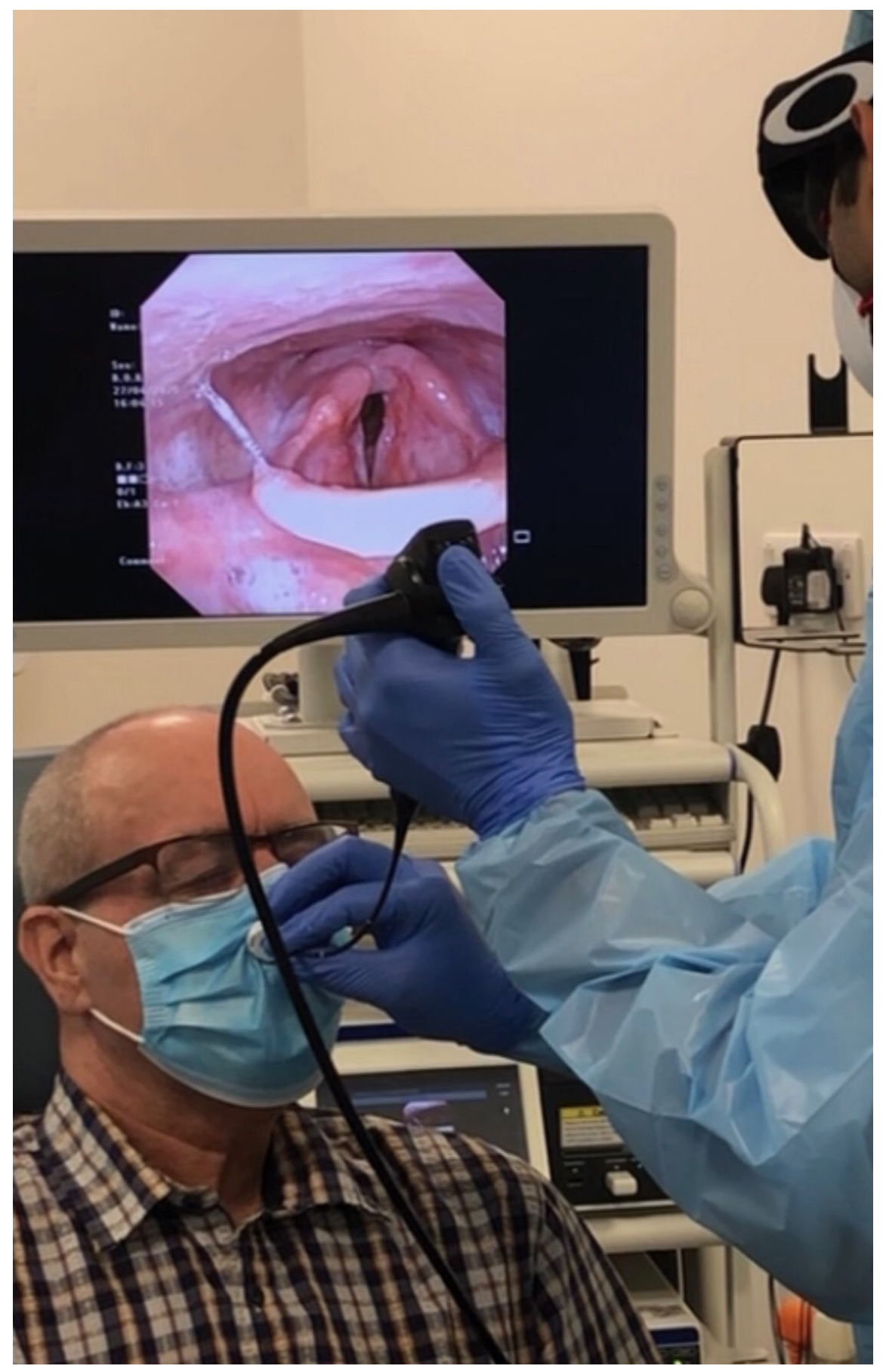




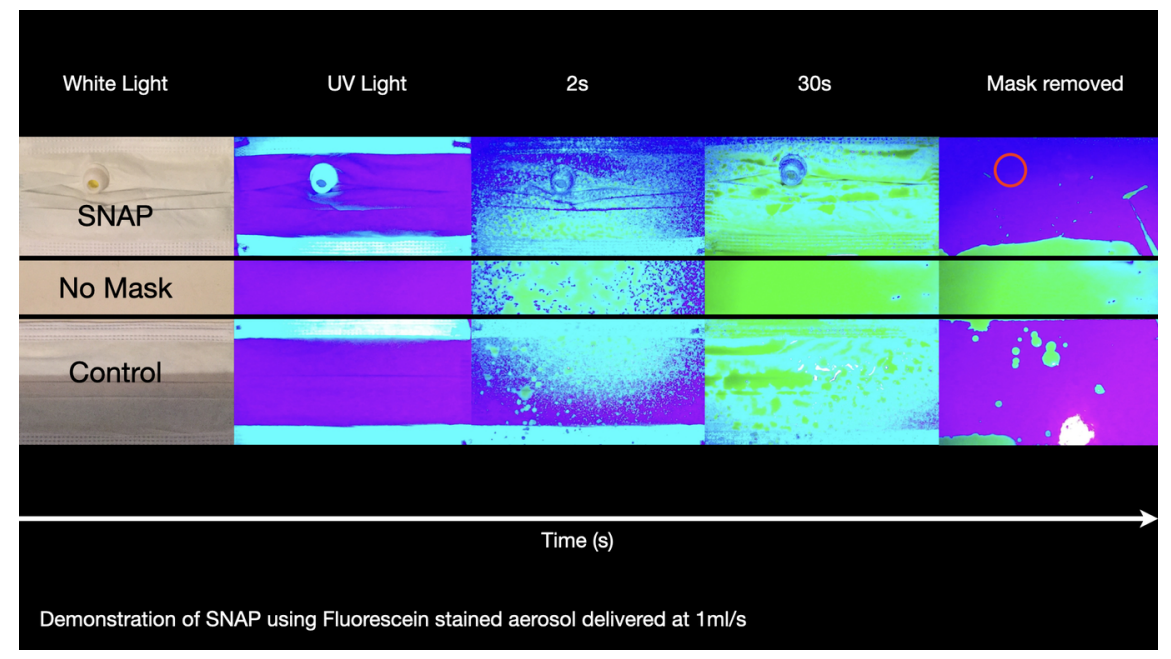

